\title{
The Shaken Baby Syndrome: An Odyssey -II Origins and Further Hypotheses-
}

\author{
Ronald H. USCINSKI*,**,*** and Dennis K. MCBRIDE*,†,† \\ *Potomac Institute for Policy Studies, Arlington, Virginia, U.S.A.; \\ ** Department of Neurosurgery, George Washington University Medical Center, \\ Washington, D.C., U.S.A.; ${ }^{* * *}$ Departments of Neurosurgery and Pediatrics, and \\ ${ }^{\dagger}$ Department of Microbiology, Georgetown University Medical Center, Washington, D.C., U.S.A.; \\ ${ }^{\dagger}$ Public Policy Institute, Georgetown University, Washington, D.C., U.S.A.
}

\begin{abstract}
Subdural bleeding in the so-called "shaken baby syndrome" is recognized as a hallmark of this syndrome, and is often noted as chronic in nature, indicating an earlier time of origin than clinical presentation. In infants and neonates, the timeframe for generating such chronic intracranial bleeding is therefore limited. Neurosurgical, obstetric, and pediatric literature all recognize the significance of birth trauma in the generation of intracranial bleeding. This possibility is explored further here, with emphasis on features peculiar to Homo sapiens predisposing to intracranial bleeding during this timeframe. Encephalization and bipedalism combine to render the infant and mother susceptible to injury at birth.
\end{abstract}

Key words: shaken baby syndrome, chronic subdural hematoma, primate, encephalization, head molding, altricial nature

\section{Introduction}

The hypothesis of causing subdural hematoma in infants solely by manual shaking without impact, although accepted in legal and public policy domains as settled, remains unsupported and in fact disproved experimentally. ${ }^{1,9,25)}$ It is now clear that if humans actually do manually shake infants, the forces required to produce acute subdural hematoma by this hypothetical mechanism cannot be generated without impact. In reviewing an extensive body of literature including published articles and clinical cases, it is noted that many of the infants supposedly shaken exhibit not acute, but chronic subdural hematomas, which themselves are known to expand and present by rebleeding without accompanying trauma. ${ }^{15-17,31)}$ It is the ultimate genesis of such hematomas, generated weeks or even months before clinical presentation, that is addressed here.

\section{Observation and Investigation}

In earlier work, ${ }^{30)}$ the chronic subdural hematoma seen in infants supposedly injured by manual shaking was addressed as an already known pathologic entity having its inception weeks or months before presentation, possibly as early as parturition. A chance observation was made of a 3-day-old infant Gorilla gorilla, holding its head erect and aware of its surroundings. This led to a more formal viewing of a video of the birth of a Pan troglodyte, behaving in similar fashion. Each animal, although a neonate, was clearly functioning at a higher level of neurologic development than its counterpart human. This prompted research wherein anatomic and physiologic comparisons were drawn between Homo sapiens not as a separate entity but rather as a member of the primate order, and other nonhuman primates. A comparison of the known factors in the process of primate parturition yielded what is considered a rationale for why birth-related intracranial bleeding may and indeed does occur in the human species.

\section{Hypotheses Regarding the Origin of Subdural Hematoma}

In the years since the establishment of the hypothesis of injuring infants by manual shaking, alternative theories to the genesis of subdural bleeding associated with infancy have been put forth. Vaccine reactions $^{3)}$ and vitamin deficiency ${ }^{6)}$ are examples. While 
such theories have plausibility and are worthy of consideration, they remain difficult to falsify, and none actually explains the chronicity of the subdural bleeding encountered. It remains possible that they exacerbate subdural bleeding already present. Yet in children as young as 2 weeks to 1 month of age, the presence of chronic subdural bleeding points to an earlier genesis. Also, while it is known that bleeding in the subdural space may occur as a result of impact, the nature of the infant skull with its deformable characteristics ${ }^{21,23)}$ would seem to render purely subdural bleeding without accompanying scalp, skull, and underlying brain pathology unlikely. Therefore, the question of the true origin of such chronic subdural hematomas remains salient.

Based on the foregoing, it is hypothesized specifically that the co-evolutionary (or sequential evolutionary) processes associated with bipedalism (requiring a narrower pelvis) and encephalization (requiring a broad pelvic opening) produced a result wherein (a) the modern fetal skull is developed to the maximal volume possible (or even beyond) in utero; (b) that the ultimate benefit of the advanced and enlarged yet relatively unmyelinated human infant brain is bought at a price, this being both maternal and infant vulnerability at childbirth.

In humans, or more specifically the human primate, the fetal head is the largest structure to pass through the maternal pelvis. The head of Homo sapiens presents essentially in the occiput anterior position, and even in normal deliveries accurate presentation, rotation, and restitution of the head must occur, as must head molding, all due to passage through the narrow pelvis of the bipedal human. Such molding of the head has been shown to occur additionally during both the first and second stages of labor, ${ }^{20)}$ and the vulvar orifice must enlarge to perhaps $12 \mathrm{~cm}$ diameter to accommodate passage of the infant cranium. This is all accomplished by uterine pressure forcing the fetus headfirst along the path of least resistance. The human uterus exerts a total of $4000-8000 \mathrm{mmHg}$ pressure to achieve complete cervical dilation alone. ${ }^{2)}$ After complete dilation, uterine contraction proceeds to propel the fetus head first, from behind, through a curved and constricted passage past the os cervix and ultimately through the stretched vulvar orifice. Under even the most optimal circumstances, it is reasonable to suggest and expect that injury to the human infant and mother, in the form of intracranial and specifically subdural bleeding in the former, and cervical or perineal laceration in the latter, may result. Due to the greater elasticity, immature ossification, and wider suture margins of the premature infant, such risk in premature or even low birth weight infants might be higher. ${ }^{18,24)}$ A common obstetrical procedure is the performance of an episiotomy to control such injuries to the mother and infant during birth. Assisted delivery by either vacuum extraction, forceps application, or caesarean section are all accepted in clinical practice, the reason usually being the infant is simply not delivering as it should.

Intracranial bleeding in the form of either subdural hematoma or parenchymal injury to the brain has long been recognized as a complication of the birth process in the human infant. In 1905, Cushing8) described subdural bleeding as a consequence of head molding at birth, and also pointed out the significance of overriding sutures, two parameters seen to this day on newborn examination assessments. While the thrust of his paper dealt with surgical treatment, the etiology of infantile subdural hematoma resulting from parturition is clear. Others ${ }^{5,7,14)}$ have further refined the sources of injury and shown that, in addition to the normally understood tearing of bridging cortical veins, subdural hemorrhaging could occur as a result of traction and tearing of the falx and of the tentorium. This was thoroughly summarized in 1964 by Hirakawa and Nakamura. ${ }^{13)}$ Subdural hematoma has also been reported anecdotally in utero. ${ }^{11,12)}$

Modern-day first world obstetrics has quite obviously reduced the incidence of severe birth trauma, but today intracranial bleeding is still recognized as a consequence of even normal uncomplicated term vaginal delivery. A comparison in fetal and maternal mortality between current first world and third world countries, and even in the U.S.A. and Japan in the first half of the 20th century, ${ }^{13,24,26)}$ would give a truer index of human birth-related injuries, and would seem to be a fruitful area for further objective research comparing human parturition with that of nonhuman primates.

Anecdotally, it has also been noted by one of the authors (R.H.U.) that a seemingly high incidence of neonatal jaundice was recorded in the medical records of children presenting later with chronic subdural bleeding. Physiologically this would make sense if a suddenly increased level of fetal hemoglobin resulting from a more than normally physiologic quantity of degenerating fetal red cells, i.e. from intracranial bleeding, were presented to a newly maturing infant liver. This area may also present a possibility for further study.

While it is supposed that subdural bleeding should reabsorb spontaneously, the known process of membrane formation leading to slow enlargement by rebleeding in some cases may result in clinical presentation weeks or even months later. This is well-known and understood in adults, and it is also 
described in infants. ${ }^{10,13)}$ The cellular structure of the subdural membrane in adults and children is identical. Should the initial subdural hematoma be of such size as to escape clinical notice at its inception and should absorption of such a hematoma be ineffective, the expansile properties of the human infant skull, enlarging in response to intracranial volume changes already occurring to accommodate the growing myelinating brain, would be expected to enlarge further to accommodate the additional mass of the subdural hematoma. This is precisely why, as a routine measure, infant head circumference is recorded during periodic neonatal visits to the pediatrician or pediatrics clinic. A disproportionate increase in head circumference raises the possibility of an occult intracranial process such as subdural hematoma. In the adult situation, although the head does not expand, an increasing accumulation of subdural fluid may accumulate in the extracranial space rendered available by a degree of cortical atrophy. With regards to the duration of the presence of chronic subdural hematoma in infancy, a true index of the duration of its existence is not clear, although it is noteworthy that the literature establishes that such subdural hematoma may be present long enough to undergo calcification. ${ }^{22)}$

\section{Humans as Primates}

Variation among primates provides insight into this hypothesis. Investigation of prehuman fossils indicates that the adult Homo sapiens brain expanded in size by a factor of 3 over the past 3 to 4 million years, ${ }^{4)}$ putting it significantly above allometric "predictions" of brain volumes and masses among mammals and in particular among primates. A simple example of pelvic/infant head ratio ${ }^{27-29)}$ illustrates the disparity between the human infant head and the maternal pelvic aperture, and a revealing portrayal of representative infant occipital frontal circumference and available maternal aperture is noted in the accompanying Fig. 1, adapted in part from Rosenberg and Trevathan. ${ }^{27)}$

While the Macaca and Hylobate species approach the maximum planar aperture, the human actually exceeds the maximum planar aperture and therefore must solve the challenge of birth passage by exploiting a multidimensional solution, to include rotation of the head and shoulders, neck flexion and extension, and in particular, head molding. The result is forcing the fetus ideally head first via uterine fundal compression and contraction through the pelvis and against an initially closed and eventually dilated cervical os with the average intensity of uterine contractions between 60 and $70 \mathrm{mmHg}$. This would be

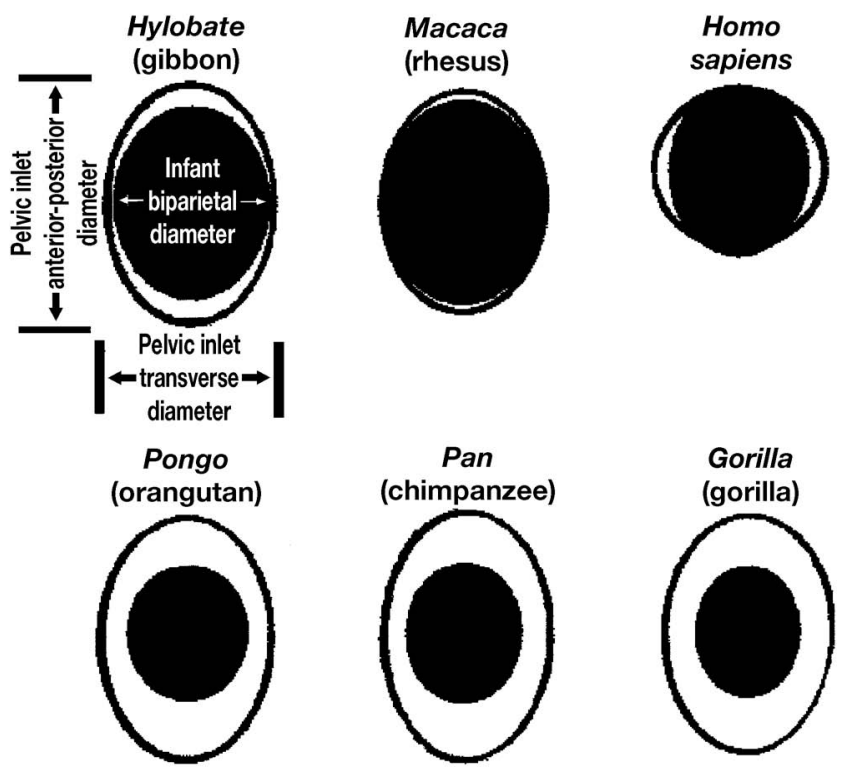

Fig. 1 Adaptation of portion of schematic by Rosenberg and Trevathan of classic diagram by Schultz illustrating relative size of maternal pelvic inlet and neonatal head in different primate species. Represented outlines are diagrammatic, but scaled so that transverse diameter of all maternal pelvic inlets are constant and all dimensions are correctly scaled relative to one another. For each species, the outlined oval represents the average maternal pelvic inlet and the black oval represents the average infant cranium. The infant cranium is oriented so that the biparietal diameter is represented as horizontal, the sagittal diameter as vertical. Note that in the Macaca and the Hylobate the dimensions of the infant cranium are only slightly smaller than the dimensions of the corresponding maternal pelvic inlet. In great apes, the pelvic inlet is relatively spacious. In humans, the infant cranium is actually longer than the anteriorposterior dimension of the pelvic inlet, requiring the head to enter the inlet facing sideways, or transversely.

expected to continue until the fetus is expelled, estimated total being between 80 and 160 uterine contractions. ${ }^{2)}$

The human infant cranium is comparatively larger than its nonhuman counterpart, due specifically to the significantly enlarged telencephalon. However, despite the larger brain, in Cushing's ${ }^{8)}$ own words, the newborn human is in actuality a "spinal animal," and lacking in the more complete myelination necessary for higher cortical function and even basic locomotion. The process of myelination, if al- 
lowed to progress to any further degree prior to birth, would render the human telencephalon so large that passage through the maternal pelvis would no longer be possible. Even under current evolutionary circumstances, it is arguable that the human birth is a compromise for the human cerebral hemispheres, which must be literally forced through parturition by head molding and cranial distortion with consequent risk of injury to relevant neuroprotective structures, and an altricial newborn. At term, myelination of only such basic tracts as the median longitudinal fasciculus and vestibular system are complete, allowing orientation of the infant head and neck in space, thereby facilitating elementary survival behavior such as rooting and suckling.

With regard to intracranial injury to the infant and perineal injury to the mother, it is not presently clear that either of the above-mentioned complications occurs in nonhuman primates. Available data ${ }^{28,29)}$ again would suggest there should be very little obstetric challenge to many or most primates. However, in those primates (all Cercopithecoidea, Hylobatidae, and all platyrrhine except howlers) wherein one or the other cranial newborn dimensions exceeds or approximates the corresponding diameter of the female pelvic inlet, abortions, miscarriages, and still births are common, and in squirrel monkeys where the cranial dimensions of newborns are the largest compared to female pelvic dimensions, infant mortality is over $50 \%,{ }^{19)}$ a finding warranting further study and analysis, particularly in light of earlier human infant mortality rates. ${ }^{13,24,26)}$ Additionally, in direct contrast to the human, nonhuman primates present in the occiput posterior position, as far as we can discern, do not rotate during fetal descent, and are born facing anteriorly. ${ }^{29)}$ Moreover, newborn nonhuman primate infants may themselves assist at delivery by use of their limbs to aid in pushing themselves out of the vulvar orifice and literally crawling across the mother's abdomen to begin suckling. ${ }^{29)}$ Finally, we have found no reports of head molding in nonhuman primates.

The altricial nature of the human infant when compared with nonhuman primates seems to indicate another dimension of compensating protection at birth. This is regular active participation by other parties (i.e., as midwifery and obstetrics) in assisting parturition. ${ }^{19)}$ Other nonhuman primates such as the chimpanzee actually isolate themselves when giving birth in order to prevent destruction of the newborn by other competing females apparently for selfpreservation of their own genetic lines, a direct opposite to human behavior.

\section{Conclusions}

It is our hope that the above observations and hypotheses provide a framework for further investigation into the antepartum and parturitional factors we believe contribute to intracranial hemorrhage in the human newborn. These observations initially stemmed from a direct concern about the misapplication of science in certain clinical and social settings, yet this in itself has provoked further investigation, transcending prior thought and assumptions on our part. In arriving at this framework, a bridge has been laid between the various disciplines of primatology, anthropology, biomechanics, and most important, medicine, in an attempt to ascertain unifying explanation for certain observed phenomena.

It is our belief that the human birth process represents an evolutionary compromise, albeit a functional one. Therefore, it cannot be overemphasized that birth injuries, both overt and occult, are a given. Any contrary position would thus a priori not only be inaccurate and scientifically unsound, but would, and perhaps already has, caused an impediment to further research and illumination of this area of human endeavor. This is not scientifically acceptable and is in fact an indirect form of intellectual, social, and legal censorship.

\section{Acknowledgments}

The authors are particularly indebted to the seminal work of Prof. Wenda Trevathan, whose insights and contributions to our understanding of primate behavior and maternal-fetal anthropology provided a uniquely solid foundation upon which to build our own study. Also of note is the illuminating paper of Hirakawa and Nakamura, whose work may have played a role greater than they could have imagined when they published it. Finally, we would like to thank Dessa Dal Porto of the Potomac Institute and Sharon Di Chiara of the Jane Goodall Institute for Primate Research (Arlington, Va., U.S.A.), for their efforts in obtaining much of the critical primate research material used in this work.

\section{References}

1) Bandak FA: Shaken baby syndrome: a biomechanics analysis of injury mechanisms. Forensic Sci Int 151: 71-79, 2005

2) Buhimschi CS, Buhimschi IA, Malinow AM, Saade GR, Garfield FR, Weiner CP: The forces of labour. Fetal and Maternal Medicine Review 14(4): 273-307, 2003 
3) Buttram HE: Shaken baby syndrome or vaccine-induced encephalitis? Medical Sentinel 6(3): 83-89, 2001

4) Cartwright J: Chapter 6, in: Evolution and Human Behavior. Cambridge, Mass, MIT Press, 2000, pp 169-175

5) Chase W: An anatomical study of subdural haemorrhage associated with tentorial splitting in the newborn. Surg Gynecol Obstet 51: 31-41, 1930

6) Clemetson CAB: Is it "shaken baby," or Barlow's disease variant? Journal of American Physicians and Surgeons 9(3): 78-80, 2004

7) Craig W: Intracranial haemorrhage in the newborn. Arch Dis Child 13: 89-134, 1938

8) Cushing $\mathrm{H}$ : Concerning surgical intervention for the intracranial hemorrhages of the newborn. Am J Med Sci 130: 563-581, 1905

9) Duhaime AC, Gennarelli TA, Thibault LE, Bruce DA, Margulies SS, Wiser R: The shaken baby syndrome. A clinical, pathological, and biomechanical study. J Neurosurg 66: 409-415, 1987

10) Guthkelch AN: Subdural effusions in infancy: 24 cases. Br Med J 1(4804): 233-239, 1953

11) Hadzikaric N, Al-Habib H, Al-Ahmad I: Idiopathic chronic subdural hematoma in the newborn. Childs Nerv Syst 22: 740-742, 2006

12) Hanigan WC, Ali MB, Cusack TJ, Miller TC, Shah JJ: Diagnosis of subdural hemorrhage in utero. Case report. J Neurosurg 63: 977-979, 1985

13) Hirakawa K, Nakamura N: [Chronic infantile subdural hematoma in infants and children]. Shoni No Seishin To Shinkei 4: 84-94, 1964 (Jpn)

14) Holland E: Cranial stress in the foetus during labour and on the effects of excessive stress on the intracranial contents; with an analysis of eighty-one cases of torn tentorium cerebelli and subdural cerebral haemorrhage. BJOG: An International Journal of Obstetrics and Gynaecology 29(4): 549-571, 1922

15) Ito H, Komai T, Yamamoto S: Fibrinolytic enzyme in the lining walls of chronic subdural hematoma. J Neurosurg 48: 197-200, 1978

16) Ito H, Yamamoto $S$, Komai $T$, Mizukoshi H: Role of local hyperfibrinolysis in the etiology of chronic subdural hematoma. J Neurosurg 45: 26-31, 1976

17) Kawakami K, Chikama M, Tamiya T, Shimamura Y: Coagulation and fibrinolysis in chronic subdural hematoma. Neurosurgery 25: 25-29, 1989

18) Kriewall TJ: Structural, mechanical, and material properties of fetal cranial bone. Am J Obstet Gynecol 143: 707-714, 1982

19) Leutenegger W: Functional aspect of pelvic morphology in simian primates. Journal of Human Evolution 3: 207-222, 1974

20) Lindgren L: The causes of foetal head moulding in labour. Acta Obstet Gynecol Scand 39: 46-62, 1960

21) Margulies SS, Thibault K: Infant skull and suture properties: measurements and implications for mechanisms of pediatric brain injury. J Biomech Eng
122: $364-371,2000$

22) McLaurin RL, McLaurin KS: Calcified subdural hematomas in childhood. J Neurosurg 24: 648-655, 1966

23) McPherson GK, Kriewall TJ: The elastic modulus of fetal cranial bone: a first step towards an understanding of the biomechanics of fetal head molding. $J$ Biomech 13: 9-16, 1980

24) Munro D: Cranial and intracranial damage in the newborn: end-result study of 117 cases. Surg Gynecol Obstet 47: 622-630, 1928

25) Prange MT, Coats B, Duhaime AC, Margulies SS: Anthropomorphic simulations of falls, shakes, and inflicted impacts in infants. J Neurosurg 99: 143-150, 2003

26) Roberts $M H$ : Intracranial hemorrhage in the newborn. JAMA 13: 280-284, 1939

27) Rosenberg K, Trevathan W: Bipedalism and human birth: The obstetrical dilemma revisited. Evolutionary Anthropology 4(5): 161-168, 1995

28) Schultz AH: Sex differences in the pelves of primates. Am J Phys Anthropol 7: 401-423, 1949

29) Trevathan WR: Human Birth. An Evolutionary Perspective. Hawthorne, NY, Aldine De Gruyter, 1987, pp 17-29

30) Uscinski RH: Shaken baby syndrome: an odyssey. Neurol Med Chir (Tokyo) 46: 57-61, 2006

31) Yamashima T, Yamamoto S, Friede RL: The role of endothelial gap junctions in the enlargement of chronic subdural hematomas. J Neurosurg 59: 298-303, 1983

Address reprint requests to: Ronald H. Uscinski, M.D., Box 538, Great Falls, VA 22066-3526, U.S.A.

e-mail: ruscinski@uscinski.net

\section{Commentary}

This presentation by Uscinski and McBride is of course of paramount relevance to medicolegal considerations on child abuse. Obviously the origin of chronic subdural haematomas in neonates is perfectly uncertain in most cases.

In the absence of any further evidence of injury or impact like bruises, broken bones, etc., the cause of a chronic subdural haematoma mostly remains pure speculation and, as the authors argue, in consideration of the anatomy of the infant's skull and brain, it appears impossible to produce a chronic subdural haematoma without impact. Also in adults, the cause of chronic subdural haematoma remains unclear in terms of evidence based medicine, as the common explanation, a bleeding from bridging veins, has never been substantiated and is rarely confirmed during operative removal. 
On the other hand, it appears plausible that injuries of the head at the time of birth are more frequent in humans than any other primates for anatomical reasons. From the data compiled in this paper, we may conclude the frequent reproach to possibly innocent parents of having violently mistreated their newborns causing chronic subdural haematomas is based on the same lack of scientific evidence and justification as medieval witch hunting.

We are grateful to the authors for reminding us not to blame anyone without adequate evidence.

Raimund FIRSCHING, M.D., L.R.C.P., M.R.C.S. Klinik für Neurochirurgie Otto-von-Guericke-Universität Magdeburg, Germany

The authors raise several points regarding shaken baby syndrome which deserve further elaboration. They correctly note that a pure shaking mechanism probably does not account for the spectrum of cranial injuries seen in this condition. The current term for the syndrome is Non-accidental Trauma, a more neutral designation that does not imply a specific mechanism of injury.

The authors also provide a comparison of the anatomical features of humans and other primates in ord- er to provide a rationale for the incidence of birthrelated brain hemorrhage. It seems plausible that certain evolutionary compromises occurred in order to allow early enough birth of human neonates before the rapid growth in head size would prevent fetal passage through the birth canal. Although this can result in birth trauma, which is well recognized, it is unclear how this relates to non-accidental trauma (or shaken baby syndrome). While birth-related subdural hematomas could be confused with subdural hematoma caused by later trauma, the other features of the syndrome such as long bone fractures, retinal hemorrhages, and soft tissue bruising do not fit into a birth mechanism. It is worth noting that subdural hematomas are not considered proof of non-accidental trauma.

The points raised in this paper highlight the many gaps in our knowledge regarding the pathogenesis of non-accidental trauma and suggest that many fruitful areas of research remain.

Nalin Gupta, M.D. and Lawrence H. PITTS, M.D. Department of Neurosurgery University of California San Francisco San Francisco, California, U.S.A. 